\title{
Modular SDN-enabled S-BVT Adopting Widely Tunable MEMS VCSEL for Flexible/Elastic Optical Metro Networks
}

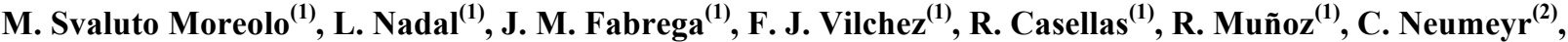 \\ A. Gatto ${ }^{(3)}$, P. Parolari ${ }^{(3)}$, P. Boffi ${ }^{(3)}$ \\ (1) Centre Tecnologic de Telecomunicacions de Catalunya (CTTC/CERCA), Castelldefels, Spain; michela.svaluto@cttc.es \\ (2) Vertilas GmbH, Daimlerstr. 11d, D-85748 Garching, Germany \\ (3) Politecnico di Milano, Dip. di Elettronica, Informazione e Bioingegneria, via G. Ponzio 34/5, 20133 Milano, Italy
}

\begin{abstract}
We propose an SDN-enabled S-BVT adopting directly-modulated tunable VCSEL with direct-detection for optical metro networks and spectrum defragmentation. We experimentally assess it over different network paths up to $185 \mathrm{~km}$ and in presence of adjacent slices.

OCIS codes: (060.4510) Optical communications; (060.4250) Networks; (140.7260) Vertical cavity surface emitting lasers.
\end{abstract}

\section{Introduction}

The migration towards a more flexible, efficient and agile paradigm of optical metro networks, characterized by stringent limitation on CAPEX/OPEX and highly dynamic and heterogeneous traffic aggregation/delivery, able to accommodate bandwidth-hungry services as well as low-rate service connections, are posing new challenges, urgently requiring low-cost and power-efficient solutions. In this context, the programmable and modular sliceable bandwidth/bitrate variable transceiver (S-BVT) represents an attractive solution, when suitably tailored for this network segment [1]. Particularly, the adaptability/reconfigurability of multiple parameters by software defined networking (SDN) combined with a modular approach, allows flexible adaptation to the path/traffic condition, with wide range granularity, while saving network resources and costs. The adoption of optoelectronic front-ends based on energy- and cost-efficient photonic technologies, appears as a crucial issue in the S-BVT design/implementation.

Tunable laser sources (TLS) with external modulation enable arbitrary tuning the optical carrier and achieving high performance. However, direct modulation (DM) of the laser source and, particularly, the adoption of VCSEL (vertical cavity surface emitting laser) allow radical reduction of transmitter cost, power consumption and footprint. VCSELs have been usually considered for short-reach, low-datarate applications at $850 \mathrm{~nm}$. Recently, their potential has been shown at $1550 \mathrm{~nm}$ with advanced modulation formats for $100 \mathrm{G}$ applications as well as for PONs and metro/access elastic optical networks (EONs) [2-4]. Multicarrier modulation, as discrete multitone (DMT) or orthogonal frequency division multiplexing (OFDM), enables spectral manipulation, implementing bit and power loading (BL/PL) at digital signal processing (DSP). This allows dynamic and flexible adaptation to traffic/channel conditions and spectrum fragmentation mitigation [1], with spectrum granularity even finer than EON $(12.5 \mathrm{GHz})$.

In this work, we propose to adopt DM widely tunable MEMS (micro-electro-mechanical system) VCSEL [3] and direct detection (DD) as cost-effective and energy-efficient building block for programmable (SDN-enabled) SBVTs able to provide improved performance/functionalities, including mitigating spectrum fragmentation, in optical metro networks. We experimentally assess that single-sideband (SSB) OFDM enables to extend the achievable reach. Furthermore, bandwidth/bitrate adaptive DSP combined with widely tunable MEMS VCSEL allows the SDN-enabled module suitably adapting to the dynamic variation of optical metro networks and filling spectral gaps for spectrum defragmentation without requiring a network re-optimization. Thanks to these features, the proposed SBVT architecture represents a promising candidate for optical metro networks with very fine spectrum granularity.

\section{SDN-enabled S-BVT system equipped with widely tunable MEMS VCSEL}

The programmable and modular S-BVT (in Fig. 1) consists of an array of $N$ BVTs and an aggregator/distributor of the generated/received multiple flows (slices). The BVT array can be composed of subsets of BVT modules (enabling a scalable pay-as-you-grow model based on pluggable units and photonic integration), whose BVTx frontend are based on either DM VCSEL or external modulator driven by TLS. The BVRx adopts DD for cost-sensitive applications. The BVT DSP allows implementing DMT or OFDM, including margin adaptive (MA) or rate adaptive (RA) BL/PL algorithms. Either double sideband (DSB, or DMT) or SSB transmission (more robust to chromatic dispersion than DMT) can be implemented. If the generated $N$ flows are aggregated by using a spectrum selective switch (SSS), it can act as both aggregator and (SSB) filter bank. The multiple slices are transmitted over the network as a single high-capacity flow or can be split into lower capacity flows routed towards independent paths [1]. The cost-effective and energy-efficient VCSEL-based module also allows suitably filling spectral gaps thanks to its tunability and adaptive narrow bandwidth, resulting in a promising technology option for mitigating the spectrum fragmentation. Furthermore, these features enable an SDN control paradigm: different parameters (related to DSP blocks, BVT front-ends and aggregator/distributor) can be suitably configured on-demand by the SDN controller, by 
C 2018 Optical Society of America. One print or electronic copy may be made for personal use only. Systematic reproduction and distribution, duplication of any material in this paper for a fee or for commercial purposes, or modifications of the content of this paper are prohibited.

https://doi.org/10.1364/OFC.2018.M1A.7

means of SDN S-BVT agents [1]. This includes DSP mode (channel estimation with uniform loading or transmission with adaptive loading), algorithm (RA/MA), target performance/bitrate, enabled BVT slice, operating wavelength, and SSS parameters such as wavelength, bandwidth and power/attenuation (per port).

For the system assessment, we set-up an array of $N=3$ BVTs (either considered two subsets), one (BVT $\mathrm{VCCEL}_{\text {, }}$ first subset) based on a widely tunable VCSEL module and the other two (BVT $\mathrm{MZM}_{\mathrm{M}}$, second subset) adopting a TLS and a MZM at the BVTx; the BVRx front-end consists of a pre-amplified DD receiver (PIN with TIA). All BVT modules are equipped with adaptive DSP enabling rate/distance and bandwidth variable transmission. The independent digital signals are converted to analog with a $64 \mathrm{GSa} / \mathrm{s}$ digital-to-analog converter (DAC); at the BVRx, the photodetected signal is captured by an oscilloscope at $100 \mathrm{GSa} / \mathrm{s}$ for demodulation and further error counting, considering a target BER of $4.62 \cdot 10^{-3}$ (HD-FEC with $7 \%$ overhead over a total of $13.4 \%$ ). The VCSEL wavelength is widely tunable within a range of up to $60 \mathrm{~nm}$. With a fine adjustment of MEMS, bias and temperature control, the VCSEL can be tuned to operate within the C-band with flexgrid granularity $(6.25 \mathrm{GHz}$ and $12.5 \mathrm{GHz}$ frequency slots). The VCSEL transfer functions measured (with a network analyzer) at $1550.12 \mathrm{~nm}$ and at the edge channels of the ITU grid $(1530.33 \mathrm{~nm}$ and $1561.42 \mathrm{~nm})$ are shown in Fig. 1b. When the VCSEL is DM with DMT adopting $\mathrm{BL} / \mathrm{PL}$ (512 subcarriers on 16GHz), the maximum bitrate, below the target BER threshold, in back-to-back (B2B) is $33.2 \mathrm{~Gb} / \mathrm{s}$ at $1550.12 \mathrm{~nm}$, and slightly lower at the edge channels $(29.8 \mathrm{~Gb} / \mathrm{s}$ and $31.3 \mathrm{~Gb} / \mathrm{s}$, respectively), according to the corresponding signal-to-noise ratio (SNR) profile. The channel state information (CSI) is retrieved at the adaptive BVRx DSP with a uniform loaded (4QAM) probing signal. The maximum bandwidth occupation is $10.5 \mathrm{GHz}$ (Fig. 1c), corresponding to a spectral occupation of two flexgrid slots of $12.5 \mathrm{GHz}$. In the case of SSB transmission, the spectral occupation is halved and the B2B bitrate below the target BER is $31.7 \mathrm{~Gb} / \mathrm{s}$ at $1550.12 \mathrm{~nm}$. This value, slightly lower than DMT, is due to the narrow SSB filtering and the VCSEL wavelength fluctuations.
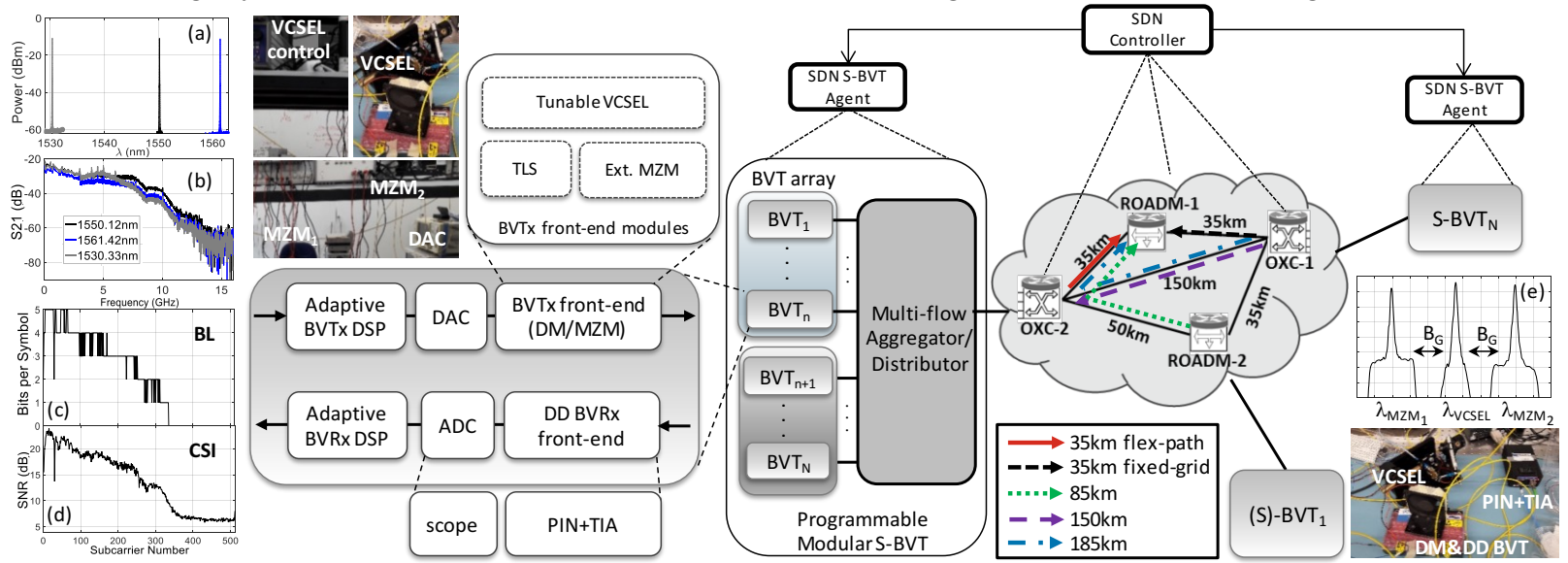

Fig. 1. SDN-enabled S-BVT system schematic; set-up with a VCSEL-based and two MZM-based BVTs (inset pictures); 4-node ADRENALINE network. Insets on the left: (a) OSA measurements of VCSEL spectrum at different wavelengths and (b) corresponding transfer functions (S21), (c) BL and (d) CSI for the VCSEL slice in B2B at $1550.12 \mathrm{~nm}$; on the right: (e) received spectra of the BVT slices with $\mathrm{B}_{\mathrm{G}}=12.5 \mathrm{GHz}$ after $35 \mathrm{~km}$.

\section{SDN-enabled S-BVT assessment for flexible and fixed-grid metro network scenarios}

We analyze the performance of the BVT $\mathrm{VCSEL}_{\mathrm{V}}$ in a 4-node network (ADRENALINE testbed) with amplified SSMF (standard single-mode fiber) links of $50 \mathrm{~km}$ and $35 \mathrm{~km}$, and a NZDSF (non-zero-dispersion-shifted fiber) link of $150 \mathrm{~km}$ (see Fig. 1). At the input/output of the network, the multi-flow is amplified by EDFA working at constant power. We first assess the case of a flexgrid path (flex-path) of $35 \mathrm{~km}$. Considering DMT, the bitrate at the target BER (RA algorithm) decreases to $21.1 \mathrm{~Gb} / \mathrm{s}$ with the tunable VCSEL set to operate at $1550.12 \mathrm{~nm}$. Similar performance is achieved at $1530.33 \mathrm{~nm}(19.8 \mathrm{~Gb} / \mathrm{s})$ and $1561.42 \mathrm{~nm}(20.5 \mathrm{~Gb} / \mathrm{s})$. By configuring the SSS as SSB filter, $28.5 \mathrm{~Gb} / \mathrm{s}$ can be achieved at $1550.12 \mathrm{~nm}$. Next, we evaluate the capacity performance of the BVT at $1550.12 \mathrm{~nm}$ below the BER threshold for DMT and SSB transmission over different ADRENALINE paths of $85 \mathrm{~km}, 150 \mathrm{~km}$ and $185 \mathrm{~km}$ (Fig. 1). SSB modulation supports bitrates $>20 \mathrm{~Gb} / \mathrm{s}$ up to a 2 -hop path of $185 \mathrm{~km}$, as shown in Fig. $2 \mathrm{a}$.

In order to assess the $\mathrm{BVT}_{\mathrm{VCSEL}}$ capability of filling spectral gaps for spectrum defragmentation, we analyze its performance in presence of adjacent slices (Fig.1e) at the varying of the guard-band $\left(\mathrm{B}_{\mathrm{G}}\right)$. The $\mathrm{BVT}_{\mathrm{VCSEL}}$ is tuned to operate at $1550.12 \mathrm{~nm}$. Each adjacent slice is generated with a $\mathrm{BVT}_{\mathrm{MZM}}$ equipped with adaptive DSP for a maximum (electrical) bandwidth of $16 \mathrm{GHz}$ (corresponding to a flexgrid channel of three $12.5 \mathrm{GHz}$ slots in case of DMT). The TLS wavelengths are set to obtain the target $\mathrm{B}_{\mathrm{G}}$, considering the slices spectral occupancy in terms of assigned flexgrid slots. For this analysis, we consider DSB transmission (DMT) over $35 \mathrm{~km}$ flex-path. Please note that we analyze the same MZM performance at different wavelengths and the aggregator is implemented with a cascade of 
(C) 2018 Optical Society of America. One print or electronic copy may be made for personal use only. Systematic reproduction and distribution, duplication of any material in this paper for a fee or for commercial purposes, or modifications of the content of this paper are prohibited.

https://doi.org/10.1364/OFC.2018.M1A.7

couplers emulating SSS insertion loss. When $\mathrm{B}_{\mathrm{G}}$ is $6.25 \mathrm{GHz}$, the total bandwidth occupation of the three slices is 112.5GHz. In this case, the $\mathrm{BVT}_{\mathrm{VCSEL}}$ supports a bitrate of $18.5 \mathrm{~Gb} / \mathrm{s}, 9.8 \%$ lower than the bitrate obtained when the adjacent slices are disabled (Fig. 2a-c). The bitrate of the $\mathrm{BVT}_{\mathrm{MZM}}$ at $1549.82 \mathrm{~nm}$ and $1550.42 \mathrm{~nm}$ is slightly lower $\left(47.9 \mathrm{~Gb} / \mathrm{s}\right.$ and $46.8 \mathrm{~Gb} / \mathrm{s}$, respectively) than $48.6 \mathrm{~Gb} / \mathrm{s}$ and $47.2 \mathrm{~Gb} / \mathrm{s}$, when the $\mathrm{BVT}_{\mathrm{VCSEL}}$ is disabled. Thus, including the cost-effective $\mathrm{BVT}_{\mathrm{VCSEL}}$ to the MZM-based BVTs subset, the overall S-BVT capacity increase is $18.2 \%$ for the same bandwidth occupation, thanks to the $\mathrm{BVT}_{\mathrm{VCSEL}}$ capability of filling spectral gaps. For $\mathrm{B}_{\mathrm{G}}=12.5 \mathrm{GHz}$ and a total bandwidth of $125 \mathrm{GHz}$ (10 slots), the crosstalk effect is negligible, as the bitrate of all slices at the target BER present rather no penalty. For this case, we analyze the $\mathrm{BVT}_{\mathrm{VCSEL}}$ capacity at the varying of its output power (adding a VOA in the set-up) with respect to adjacent slices power. The results in Fig. 2d show that the capacity is halved for $6 \mathrm{~dB}$ relative power. Finally, when $\mathrm{B}_{\mathrm{G}}$ is zero and the total bandwidth occupation is $100 \mathrm{GHz}$ (Fig. 2e), the $\mathrm{BVT}_{\mathrm{VCSEL}}$ bitrate decreases to $14.5 \mathrm{~Gb} / \mathrm{s}(29.3 \%$ penalty also due to the VCSEL wavelength fluctuation within a narrow bandwidth). The adjacent slices are less affected (also for their effective bandwidth occupation, enabling optimized $\mathrm{BL} / \mathrm{PL}$ performance) given a bitrate of $47.5 \mathrm{~Gb} / \mathrm{s}$ and $48.1 \mathrm{~Gb} / \mathrm{s}$, at $1549.87 \mathrm{~nm}$ and $1550.37 \mathrm{~nm}$, respectively. This last result shows that the SSS of ADRENALINE flex-path is acting as vestigial sideband (VSB) filter, for the slice at $1550.37 \mathrm{~nm}$ (slightly shifted towards the edge of the assigned ADRENALINE channel slots). If the number of assigned slots is increased to $10(125 \mathrm{GHz})$ the obtained bitrate is $46.7 \mathrm{~Gb} / \mathrm{s}$ (in line with the other slice penalty). The minimum bitrate without $(\mathrm{w} / \mathrm{o}) \mathrm{B}_{\mathrm{G}}$ of the $\mathrm{BVT}_{\mathrm{MZM}}$ at $1550.12 \mathrm{~nm}$, with the $\mathrm{BVT}_{\mathrm{VCSEL}}$ and the other $\mathrm{BVT}_{\mathrm{MZM}}$ as adjacent slices, is $45.1 \mathrm{~Gb} / \mathrm{s}$. In any case, an overall S-BVT capacity increase is achieved enabling the BVT $\mathrm{VCSEL}_{\text {. }}$

To compare the obtained results over a flexgrid path (with SSS at the network nodes) with a fixed-grid scenario, we analyze the case $w / 0 \mathrm{~B}_{\mathrm{G}}$ over a $35 \mathrm{~km}$ fixed-grid ADRENALINE link (equipped with standard $100 \mathrm{GHz}$ arrayed waveguide gratings). As shown in Fig. 2f (OSA measurements), the BVT $\mathrm{MZM}_{\mathrm{M}}$ slices are distorted by the fixed-grid channel profile (VSB filtering). This is evidenced by a bitrate increase of the $\mathrm{BVT}_{\mathrm{MZM}}$ at $1550.37 \mathrm{~nm}$ up to $55.3 \mathrm{~Gb} / \mathrm{s}$.

The SDN controller suitably configures the S-BVT, setting each BVT active slice by means of an SDN agent that maps high level configuration commands, sent via a REST interface, to low level hardware-specific instructions. Fig. 2g shows a configuration enabling the $\mathrm{BVT}_{\mathrm{VCSEL}}$ at $1550.12 \mathrm{~nm}$, in adaptive loading mode with RA algorithm and $9 \mathrm{~dB}$ gap for successful transmission at the target BER with HD-FEC over the selected path.
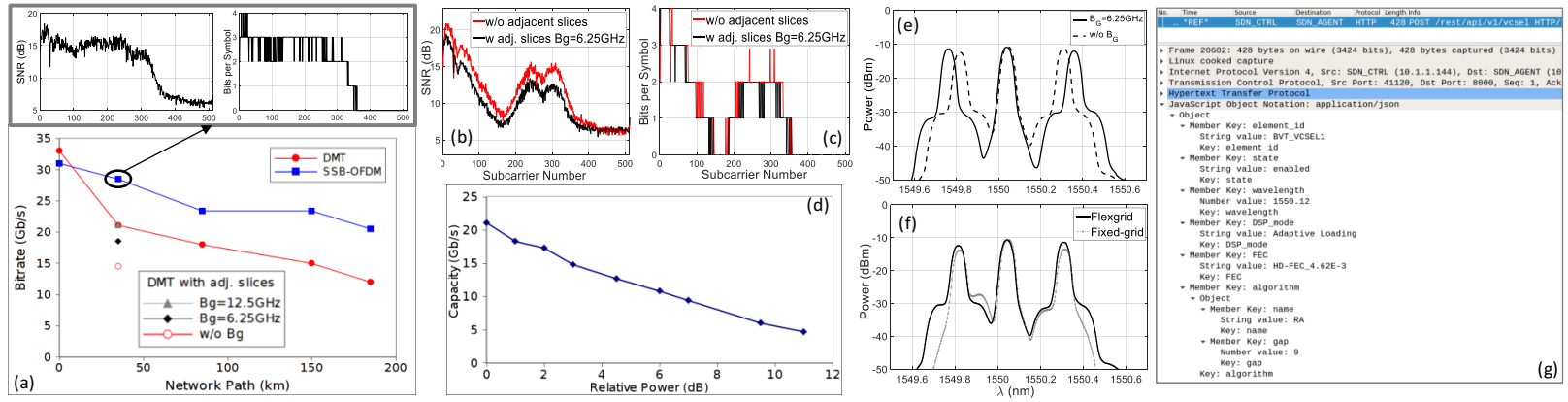

Fig. 2. (a) BVT $\mathrm{VCSEL}_{\mathrm{V}}$ bitrate vs network paths for DMT, SSB and at the varying of $\mathrm{B}_{\mathrm{G}}$; inset (a), (b), (c) CSI and BL (35km flex-path). Capacity performance vs relative power $(\mathrm{d})$. Received S-BVT spectra at the varying of $\mathrm{B}_{\mathrm{G}}(\mathrm{e})$ and grid (f). Configuration of $\mathrm{BVT}_{\mathrm{VCSEL}}$ parameters $(\mathrm{g})$.

\section{Conclusions}

We propose to adopt DM widely tunable MEMS-VCSEL, as cost-effective and energy-efficient building block, for the implementation of programmable (SDN-enabled) and modular S-BVT suitable for optical metro network applications. We experimentally assessed the proposed module in the ADRENALINE network, achieving up to $33 \mathrm{~Gb} / \mathrm{s}$ bitrate with DMT (B2B) and above $20 \mathrm{~Gb} / \mathrm{s}$ up to $185 \mathrm{~km}$ 2-hop path with SSB-OFDM. From the analysis in presence of adjacent slices, negligible bitrate penalty has been found for a $\mathrm{B}_{\mathrm{G}}$ of $12.5 \mathrm{GHz}$, enabling an S-BVT capacity increase even for lower value of $\mathrm{B}_{\mathrm{G}}$. The $\mathrm{S}-\mathrm{BVT}$ can be used in both flexible and fixed-grid scenarios (envisioning a soft-migration), even if the latter limits the performance of its advanced features. Thanks to the adaptive DSP and the SDN-enabled dynamic reconfiguration of multiple parameters, the proposed architecture represents also a promising solution to cope with spectrum fragmentation, filling spectral gaps with fine granularity.

This work has been partially supported by the EU PASSION Project (780326) and the Spanish DESTELLO project TEC2015-69256-R.

\section{References}

[1] M. Svaluto Moreolo et al. "SDN-enabled Sliceable BVT Based on Multicarrier Technology for Multi-Flow Rate/Distance and Grid Adaptation," Journal of Lightwave Technology, 34, 8, 1516-1522 (2016).

[2] C. Xie, et al., "Single VCSEL 100-Gb/s short reach system using discrete multi-tone modulation and direct detection," in OFC 2015 , Tu2H.2.

[3] C. Wagner et al., "26-Gb/s DMT Transmission Using Full C-Band Tunable VCSEL for Converged PONs," PTL 29, 17, 1475 - 1478 (2017).

[4] L. Nadal, et al., "Transparent Service Delivery in Elastic Metro/Access Networks with Cost-Effective Bandwidth Variable Transceivers," in ICTON 2017, Girona (Spain), July 2017. 